\title{
A New Retrieval Algorithm of the Thermal Infrared Optical Depth of Dust based on the Combined CALIOP and IIR Observations
}

\author{
JIANYU ZHENG ${ }^{1}$, ANNE GARNIER ${ }^{2}$, ZHIBO ZHANG ${ }^{1}$ \\ AND HONGBIN YU ${ }^{3}$ \\ ${ }^{1} \mathrm{UMBC}$ \\ ${ }^{2} \mathrm{SSAI}$ \\ ${ }^{3}$ NASA Goddard Space Flight Center \\ Presenting Author: zheng3@umbc.edu
}

Mineal dust significantly affects both shortwave solar (SW) and longwave thermal infrared (LW) radiation, which however remains uncertain. Although dust optical properties and cooling effect at the top of atmosphere (TOA) in SW range have been extensively studied for decades, dust properties and warming effect in LW range have been understudied. In this study, we develop a new way to retrieve dust optical depth in the thermal infrared $\left(\mathrm{DOD}_{\mathrm{TIR}}\right)$ by integrating the Infrared Radiometer (IIR) and the Cloud-Aerosol Lidar with Orthogonal Polarization (CALIOP), both onboard CALIPSO. The IIR provides brightness temperature (BT) observations in three TIR bands centered at $8.65,10.6$ and $12.05 \mu \mathrm{m}$, respectively. The difference between dust-laden BT and dust-free BT (dBT) is the signal for TIR absorption optical depth. Meanwhile, CALIOP provides the accurate vertical location of dust and its optical depth (DOD) at $532 \mathrm{~nm}$. By using in situ measured scattering properties of dust and the CALIOP dust extinction profiles, as well as atmospheric profiles, we estimate dust-laden and dust-free BT and hence dBT with the fast radiative transfer model using discrete ordinated method (FASDOM). By using look-up tables of dBT as a function of $\mathrm{DOD}_{\mathrm{TIR}}$, we retrieve $\mathrm{DOD}_{\mathrm{TIR}}$ based on IIR observed $\mathrm{BT}$ and estimated dust-free BT . In addition, the impact of the radiometric and ancillary data uncertainty on $\mathrm{DOD}_{\mathrm{TIR}}$ retrieval will be evaluated by using the linear perturbation method. 